\title{
Assessing Natural Forest Conservation Using Diameter Size Class Distributions in Pakistan
}

\author{
Jamal Hussain ${ }^{1,2 *}$, Muhammad Akbar ${ }^{3}$, Shaukat Ali ${ }^{2}$, Zhou Kui ${ }^{1}$, Ghulam Raza ${ }^{4}$, \\ Anwar Khan', Muhammad Zafar ${ }^{2}$, Nizami Moazzam Syed ${ }^{5}$, Sujjad Hyder², \\ Alamdar Hussain ${ }^{4}$, Faisal Hussian ${ }^{6}$ \\ ${ }^{1}$ The West Center for Economics Research, Southwestern University of Finance and Economics, 55, \\ Guanghuacun Street, Qingyang District, Chengdu, Sichuan, P. R. China, 610074 \\ ${ }^{2}$ Department of Environmental Science, Karakoram International University Main Campus University Road \\ Gilgit (15100) Pakistan \\ ${ }^{3}$ Wildlife Conservation Society, Husain Abad Colony, Jutial Gilgit, Gilgit-Baltistan, Pakistan, Pakistan \\ ${ }^{4}$ Department of Biological Science, University of Baltistan, Skardu, Gilgit-Baltistan, Pakistan \\ ${ }^{5}$ Department of Forestry, Karakoram International University Main Campus University Road Gilgit (15100) Pakistan \\ ${ }^{6}$ Post Doctorate Fellow, Institute of Nature and Environmental Technology, Kanazawa University, \\ Kakuma-mati, Kanazawa-920-1192, Japan
}

Received: 25 October 2018

Accepted: 12 January 2019

\begin{abstract}
Forests of the Naltar Valley in Pakistan are constantly threatened by natural and anthropogenic disturbances. The study examines the natural and human-induced disturbance factors that determine natural forest conservation and management in a dry temperate mountainous region. To better assess anthropogenic disturbance factors, we randomly selected 26 stands of the forest by using the point-centered quarter (PCQ) method of Cottam and Curtis to collect the information about tree species during the summer season between July to September 2015. Results show that there is no ideal size class distribution of tree species noticed in selected sample stands. Gaps in small, middle, large and extra-large classes were observed in different locations representing noticeable natural (i.e., sliding, forest fire, flood, soil erosions, earthquakes) and anthropogenic (i.e., illegal cutting, looping, tapering, burning, and grazing) disturbances. To conserve biodiversity, it is therefore essential to introduce social forestry, preventing illegal anthropogenic activities, and provide alternative livelihood options for the inhabitant to reduce anthropogenic disturbance on these forests. This study would be helpful for exposing
\end{abstract}

*e-mail: jamal.hussain51214@gmail.com hussain.jamal@smail.swufe.edu.cn 
the present status and future trends of forest tree species, as well as more awareness about conservation of forests in the region by conducting a similar study in the remaining forested areas in Gilgit-Baltistan.

Keywords: anthropogenic disturbance, biodiversity, livelihood, Point centered quarter, Pakistan

\section{Introduction}

People have long exploited resources from all forest habitat, but the more lasting anthropogenic activities have been found in temperate forests [1]. The dry temperate mountainous regions are globally unique and these regional forests help as the world's primary source of firewood and timber products [2]. Forests provide environmental services to the society, but unfortunately in Pakistan's resting forests resources are fragile. Regardless of the government's current desperate need to increase forest cover since 1955, few practical advancements have been made. Indeed, natural forest conservation levels have been persistent at a disturbing rate due to such a situation making Pakistan one of the worst countries in the rest of the world to lose its forest resources in the upcoming 30 years [3]. The conservation of forests is vital as plants absorb $\mathrm{CO}_{2}$ and produce $\mathrm{O}_{2}$ during photosynthesis [4].

Studies of diameter structure of forest stands will capture better assessment of creating each diameter size class, and it has a vital role in a stand's production and growth [5]. For better decision making and sustainable forest management, evidence on existing diameter size class distribution of a forest stand lets us forecast of forest trends [6]. Few studies have looked at the diameter breast height of dry forest stands and species $[7,8]$. The diameter distributions often help to illustrate stand structure and interpret future growth potential of a stand. Stand structure is usually considered with the frequency distribution of stems by size, and diameter breast height [9]. The estimation of the diameter distribution of a stand is essential to forest managers for assessing forest resources and planning the future [10].

The importance of the diameter size class distribution is elaborated upon from some moist temperate area of Pakistan by Siddiqui et al. [11]. The study of diameter size class distributions of trees highlights the potential of alternative models for predictions that suitably support forest conservation and management [12, 13]. Diameter distribution and the related statistical model can play an essential role in some forest-related issues, including forestry and being helpful for the better management practices of any forest [14]. Diameter size class distribution of forests provides a better image of current status and future trends of any forested area $[8,15]$. The diameter distribution is one of the keys to forest management and is used for decision-making in harvesting stages and silvicultural practices in many countries [16].
Plant species provide beautiful landscape for any area - especially natural forests, which are very important for the beautiful landscaping in any field [17]. Natural forest is essential for recreational activities, and most developing countries invest in protecting the natural forest to utilize only recreation, and temporal and spatial proposals are vital to validate the results of any research $[18,19]$. In this way, the value of forest conservation is considered to be very important for the tourism point of view as the forest is one of the significant components of natural parks [20-23]. The decay of plants is one of the most significant sources of soil nutrients as various essential element concentrations is directly associated with the primary producer [24]. On the other hand, environmental pollution caused the health of plants and using the raw materials of living plants, environmental pollution can be detected as the plants observed the nutrients in their bodies, so the forest is significant for analyzing the heavy metals concentration in any area [25-27]. Temporal and spatial studies are essential to authenticate the results of any research [28], so it is recommended to collect data on ground level to confirm the GIS-based studies [29-31].

At present, however, natural forest conservation using diameter-size class distributions in still is infancy in the existing literature's insights. Further research effort can provide more meaningful outcomes and open new prospects for academicians and practitioners. Hence, this study aims to investigate the diameter size class structure of Naltar Valley Forest and to select appropriate distributions by using statistic tools for the forest stand and the individual tree species. This research will be helpful for understanding the anthropogenic and natural disturbances of forest better and provide policy implications for the conservation and management of natural forests.

\section{Materials and Methods}

\section{Description of Study Area}

The Naltar Valley study area is located between $36^{\circ} 145$ to $36^{\circ} 223$ north latitude $\mathrm{N}$ and $74^{\circ} .108$ to $74^{\circ} .230$ east longitude E, covering a total area of 27,206 ha within the Karakoram highlands. It lies very close to Nagar District in the Karakoram Range of northern Pakistan, and $40 \mathrm{~km}$ from the Gilgit city. From the Hunza river a narrow, steep area starts, which ends up in the rocky terrain of Naltar. 


\section{Sampling}

\section{Data Collection}

The point-centered quarter (PCQ) method of Cottam and Curtis [32] has been used in various studies, for example, Ahmed and Shaukat [33], and Siddiqui [11]. In each stand, 20 points were taken at 20-meter intervals. For this, a cross made by iron was used as PCQ to collect information about tree species. The data was collected during the summer between July to September 2015. A total of 26 stands were studied from the study area (Naltar Bala and Naltar Pain) during this field sampling.

\section{Identification of Plants}

Plants samples were obtained from the field and were transferred on Herbarium sheets in the lab for identification followed by the flora of Pakistan [34].

\section{Determining Diameter Size Classes}

Diameters at breast height (Dbh) of each tree species in a stand were taken using the Dbh tap and divided into $(10 \mathrm{~cm}$ Dbh) 14 size classes, and size structures of trees were determined using the MS Excel 2016 Package. Furthermore, in each stand, size classes divided into four categories, i.e., small size classes $(10$ to $30 \mathrm{~cm}$ Dbh), middle size classes (40 to $60 \mathrm{Dbh} \mathrm{cm}$ ), and large size classes (70 to $90 \mathrm{~cm}$ Dbh) and above $(90 \mathrm{~cm} \mathrm{Dbh})$ extra-large size classes following the studies $[8,15,33$, $35]$.

\section{Statistical Analysis}

After collection of data we fed it into soft form and analyzed it using MS Excel 2016. The findings are represented in both tables and graphs. Frequency, density, basal area, abundance and importance value index (IVI) of plant species were calculated following Mueller-Dombois and Ellenberg [36] and Ahmed and Shaukat [33]. The basal area of each tree species was calculated as $\mathrm{B} . \mathrm{A}=\mathrm{Pi}(1 / 2 \mathrm{Dbh})^{2}$. The importance value index (IVI) of trees were calculated by summing the relative frequency, relative density and relative dominance for trees and shrubs. Importance value index was calculated from the values of relative frequency, relative basal area, and relative density. Table 1 depicts the formulas used to calculate the density, frequency, basal area, and importance value index (IVI) and their corresponding values.

\section{Collection of Geographical Coordinates}

Aspects and elevation of each stand were recorded using a GPS device. Slope angle was measured by the clinometers (Suunto Height and Normal slope meter PM-5/1520 PC).

\section{Results and Discussion}

In the study area a total of four tree species were identified. Among them, three species (i.e. Pinus wallichiana, Picea smithiana and Juniperus excelsa) were gymnospermic, while one of them, Betula utilis,

Table 1. Formulae used to analyze the parameters of the study.

\begin{tabular}{|c|c|c|c|}
\hline S.No & Parameters & Symbols & Formulae \\
\hline 1 & Mean Distance & $d_{1}$ & $\{\Sigma$ of all distances $/ \Sigma$ of all points taken $\}$ \\
\hline 2 & Mean Area & $\left(d_{1}\right)^{2}$ or $M$ & $\left\{\right.$ Square of Mean Distance or $\left.\left(\mathrm{d}_{1}\right)^{2}\right\}$ \\
\hline 3 & Stand Density ha ${ }^{-1}$ & $\mathrm{D}_{9}$ & $\{10000 / \mathrm{M}\}-------1$ ha $=10000$ meters \\
\hline 4 & Frequency & $\mathrm{F}_{1}$ & No. of a Quadrates in which a specie occurs $\div$ Total No. of Quadrates *100 \\
\hline 5 & Relative Frequency & $\mathrm{F}_{3}$ & $\mathrm{~F}_{1}$ of a specie $\div \Sigma \mathrm{F}_{1}$ of all specie* 100 \\
\hline 6 & Relative Density & $\mathrm{D}_{3}$ & No. of individual of a specie $\div$ Total No. of individual of All aspects i.e.*100 \\
\hline 7 & $\begin{array}{c}\text { Average Basal Area of } \\
\text { a specie in } \mathrm{cm}^{2}\end{array}$ & A.B.A & Total B.A of a specie $\div$ Total No. of individual of a specie \\
\hline 8 & $\begin{array}{c}\text { Relative Basal Area of } \\
\text { a specie in } \mathrm{cm}^{2}\end{array}$ & $\mathrm{~B}_{3}$ & B.A of a specie $\div$ B.A of all species $* 100$ \\
\hline 9 & Density ha ${ }^{-1}$ of a specie & & $\{\mathrm{D} 3$ of a specie*D9 $\div 100\}$ \\
\hline 10 & $\begin{array}{c}\text { Basal Area meter square per } \\
\text { hectare }\end{array}$ & B.A m²ha ${ }^{-1}$ & density per ha of a specie*Average B.A of same specie $\div 10000$ \\
\hline 11 & Important Value Index & IVI & $\left\{\mathrm{F}_{3}+\mathrm{D}_{3}+\mathrm{B}_{3}\right\} \div 3 ”$ \\
\hline
\end{tabular}

Reference: Mueller-Dombois and Ellenberg [36] and Ahmed and Shaukat [33].” 
Table 2. Study area location, elevation, GPS readings and slope description.

\begin{tabular}{|c|c|c|c|c|c|c|c|c|}
\hline STD & Name of forest & Location & Lat. (N) & Long. (E) & Ele. m & Aspect & Slope (o) & Canopy \\
\hline 1 & Plate & Skiing area & 36.154 & 74.186 & $3008 \mathrm{~m}$ & NE & $45^{\circ}$ & Close \\
\hline 2 & Ishkoman & Skiing area & 36.146 & 74.177 & $3524 \mathrm{~m}$ & $\mathrm{NE}$ & $45^{\circ}$ & Close \\
\hline 3 & Danoe Ghotom & Nagarah & 36.145 & 74.181 & $3357 \mathrm{~m}$ & NW & $45^{\circ}$ & Open \\
\hline 4 & Chimerso-A & Chimerso & 36.189 & 74.147 & $3008 \mathrm{~m}$ & SE & $25^{\circ}$ & Open \\
\hline 5 & Chimerso-B & Chimers & 36.182 & 74.151 & $2988 \mathrm{~m}$ & SE & $15^{\circ}$ & Close \\
\hline 6 & Shafda Nada & Kon-Kae & 36.168 & 74.162 & $3130 \mathrm{~m}$ & SE & $55^{\circ}$ & Moderate \\
\hline 7 & Koto-A & Naltar Lake & 36.222 & 74.108 & $3289 \mathrm{~m}$ & SE & $10^{\circ}$ & Open \\
\hline 8 & Koto-B & Koto & 36.223 & 74.110 & $3247 \mathrm{~m}$ & SE & $10^{\circ}$ & Close \\
\hline 9 & Koto-C & Naltar Bala & 36.223 & 74.113 & $3219 \mathrm{~m}$ & SE & $10^{\circ}$ & Close \\
\hline 10 & Bangala-A & Naltar Bala & 36.221 & 74.115 & $3202 \mathrm{~m}$ & SE & $05^{\circ}$ & Close \\
\hline 11 & Bangala-B & Naltar Bala & 36.218 & 74.117 & $3188 \mathrm{~m}$ & SE & $05^{\circ}$ & Close \\
\hline 12 & Besha Geri-A & Naltar Bala & 36.212 & 74.128 & $3170 \mathrm{~m}$ & $\mathrm{NE}$ & $12^{\circ}$ & Moderate \\
\hline 13 & Besha Geri-B & Naltar Bala & 36.211 & 74.131 & $3163 \mathrm{~m}$ & NE & $15^{\circ}$ & Close \\
\hline 14 & Besha Geri-C & Naltar Bala & 36.209 & 74.133 & $3133 \mathrm{~m}$ & $\mathrm{NE}$ & $10^{\circ}$ & Close \\
\hline 15 & Besha Geri-D & Naltar Bala & 36.206 & 74.137 & $3103 \mathrm{~m}$ & $\mathrm{NE}$ & $05^{\circ}$ & Close \\
\hline 16 & Lower Besha Geri & Naltar Bala & 36.199 & 74.142 & $3090 \mathrm{~m}$ & $\mathrm{NE}$ & $20^{\circ}$ & Close \\
\hline 17 & Faingi & Naltar Pain & 36.116 & 74.222 & $3072 \mathrm{~m}$ & SE & $50^{\circ}$ & Moderate \\
\hline 18 & Faingi Khurrung-A & Naltar Pain & 36.116 & 74.223 & $3083 \mathrm{~m}$ & SE & $45^{\circ}$ & Moderate \\
\hline 19 & Faingi Khurrung-B & Naltar Pain & 36.116 & 74.224 & $3092 \mathrm{~m}$ & SE & $45^{\circ}$ & Moderate \\
\hline 20 & Faingi Khurrung-C & Naltar Pain & 36.116 & 74.225 & $3075 \mathrm{~m}$ & SE & $45^{\circ}$ & Open \\
\hline 21 & Faingi Khurrung-D & Naltar Pain & 36.115 & 74.230 & $3152 \mathrm{~m}$ & SE & $40^{\circ}$ & Close \\
\hline 22 & Faingi Harchingah & Naltar Pain & 36.207 & 074.125 & $3161 \mathrm{~m}$ & SE & $40^{\circ}$ & Close \\
\hline 23 & Harchin Gah & Naltar Pain & 36.185 & 074.134 & $3121 \mathrm{~m}$ & SE & $35^{\circ}$ & Close \\
\hline 24 & Hodan Khori-A & Naltar Pain & 36.206 & 074.118 & $3145 \mathrm{~m}$ & SE & $45^{\circ}$ & Close \\
\hline 25 & Hodan Khori-B & Naltar Pain & 36.202 & 074.126 & $3139 \mathrm{~m}$ & SE & $35^{\circ}$ & Close \\
\hline 26 & Toli Buz & Naltar Pain & 36.210 & 074.113 & $3182 \mathrm{~m}$ & SE & $40^{\circ}$ & Close \\
\hline
\end{tabular}

belongs to an angiosperm family. Diameter size class structure of each stand is presented in Fig. 1. Every forest stand is briefly discussed with sites, locations, environmental characteristics (i.e., elevation, slope angles, exposures) and graphical information (i.e., latitude and longitude) in Table 3. Physical and other observational characteristics of forests stands are also given. The size-class structure of all stands is detailed and discussed below.

This study showed that Pinus wallichiana is the first dominant tree species in 3 stands and as a pure stand present in 08 sides, while in 4 stands it is distributed as a second dominant tree. Similarly, Picea smithiana is the second dominant tree species in 2 stands and this species present in 4 stands as pure, while in 10 stands it is distributed as a first dominant tree. Betula Utilis was present in 2 stands as a pure stand and in 4 stands as a second plentiful species. Juniperus excelsa appeared as second dominant only in stand No. 16 (Lower Besha Giri) Naltar Bala, with 82.30 stems ha-1. Most of the forests show gaps in earlier size classes with low density. This shows livestock overgrazing and cutting of young trees in which it is hard for young seedlings to survive. This situation may be overcome by promoting seedling regeneration in these areas, but many stands also show gaps in large size classes, indicating extensive cutting.

The distribution of each tree's individuals vs. size classes are investigated, and as a result it is observed that Pinus wallichiana was found as a dominant tree species having $170.6 \pm 30.77$ mean density-ha ${ }^{1-}$ in the entire forested area, securing $1^{\text {st }}$ dominant in eleven stands and $2^{\text {nd }}$ dominant in five stands, while this species was never noticed as the $3^{\text {rd }}$ species in any stands (Table 4). A similar study done by [37] in the area of 
Table 3. Phytosociological attributes, rank, and total values of 26 stands in Naltar Valley Gilgit, Pakistan.

\begin{tabular}{|c|c|c|c|c|c|c|c|c|}
\hline \multirow{2}{*}{$\begin{array}{l}\text { Location } \\
\text { and sites }\end{array}$} & \multirow{2}{*}{ Species Name } & \multicolumn{4}{|c|}{ Attributes of Phytosociology } & \multirow{2}{*}{ Rank } & \multicolumn{2}{|c|}{ Total values } \\
\hline & & R.F & R.D & R.B.A & IVI & & $\mathrm{D} / \mathrm{ha}^{-1}$ & $\mathrm{BAm}^{2} \mathrm{ha}^{-1}$ \\
\hline \multicolumn{9}{|c|}{ Naltar Bala } \\
\hline Stand 1 & Picea smithiana & $100 \%$ & $100 \%$ & $100 \%$ & 100 & Pure & 60.83 & 20.60 \\
\hline Stand 2 & Betula utilis & $100 \%$ & $100 \%$ & $100 \%$ & 100 & Pure & 126.71 & 11.11 \\
\hline Stand 3 & Picea smithiana & $100 \%$ & $100 \%$ & $100 \%$ & 100 & Pure & 93.91 & 32.40 \\
\hline \multirow{2}{*}{ Stand 4} & Pinus wallichiana & $80 \%$ & $94 \%$ & $90 \%$ & 88 & $1^{\mathrm{st}}$ & 220.20 & 27.33 \\
\hline & Picea smithiana & $20 \%$ & $6 \%$ & $10 \%$ & 12 & $2^{\text {nd }}$ & 14.06 & 0.20 \\
\hline Stand 5 & Pinus wallichiana & $100 \%$ & $100 \%$ & $100 \%$ & 100 & Pure & 232.99 & 29.87 \\
\hline \multirow{2}{*}{ Stand 6} & Picea smithiana & $80 \%$ & $94 \%$ & $91 \%$ & 88.33 & $1^{\text {st }}$ & 150.10 & 18.07 \\
\hline & Pinus wallichiana & $20 \%$ & $06 \%$ & $09 \%$ & 11.67 & $2^{\text {nd }}$ & 9.58 & 0.12 \\
\hline Stand 7 & Betula utilis & $100 \%$ & $100 \%$ & $100 \%$ & 100 & Pure & 56.24 & 3.54 \\
\hline Stand 8 & Pinus wallichiana & $100 \%$ & $100 \%$ & $100 \%$ & 100 & Pure & 258.80 & 39.47 \\
\hline Stand 9 & Pinus wallichiana & $100 \%$ & $100 \%$ & $100 \%$ & 100 & Pure & 286.94 & 44.71 \\
\hline Stand 10 & Pinus wallichiana & $100 \%$ & $100 \%$ & $100 \%$ & 100 & Pure & 307.41 & 62.40 \\
\hline \multirow{2}{*}{ Stand 11} & Pinus wallichiana & $\begin{array}{l}69 \% \\
31 \% \\
\end{array}$ & $\begin{array}{l}85 \% \\
15 \% \\
\end{array}$ & $\begin{array}{l}83 \% \\
17 \% \\
\end{array}$ & $\begin{array}{l}79 \\
21 \\
\end{array}$ & $\begin{array}{l}1^{\text {st }} \\
2^{\text {nd }}\end{array}$ & $\begin{array}{c}151.60 \\
26.76 \\
\end{array}$ & $\begin{array}{c}23.26 \\
0.82\end{array}$ \\
\hline & Picea smithiana & & & & & & & \\
\hline Stand 12 & Pinus wallichiana & $100 \%$ & $100 \%$ & $100 \%$ & 100 & Pure & 159.41 & 18.20 \\
\hline Stand 13 & Pinus wallichiana & $100 \%$ & $100 \%$ & $100 \%$ & 100 & Pure & 293.43 & 27.35 \\
\hline Stand 14 & Pinus wallichiana & $100 \%$ & $100 \%$ & $100 \%$ & 100 & Pure & 331.13 & 44.70 \\
\hline Stand 15 & Pinus wallichiana & $100 \%$ & $100 \%$ & $100 \%$ & 100 & Pure & 266.67 & 50.96 \\
\hline \multirow{2}{*}{ Stand 16} & Pinus wallichiana & $57 \%$ & $68 \%$ & $88 \%$ & 71 & $1^{\text {st }}$ & 174.9 & 28.53 \\
\hline & Juniperus excels & $43 \%$ & $32 \%$ & $12 \%$ & 29 & $2^{\text {nd }}$ & 82.30 & 1.66 \\
\hline \multicolumn{9}{|c|}{ Naltar Pain } \\
\hline \multirow{2}{*}{ Stand 17} & Picea smithiana & $91 \%$ & $97 \%$ & $98 \%$ & 95.33 & $1^{\mathrm{st}}$ & 118.07 & 14.22 \\
\hline & Pinus wallichiana & $09 \%$ & $03 \%$ & $02 \%$ & 4.67 & $2^{\text {nd }}$ & 11.70 & 0.07 \\
\hline \multirow{2}{*}{ Stand 18} & Picea smithiana & $95 \%$ & $99 \%$ & $99 \%$ & 97.67 & $1^{\text {st }}$ & 126.58 & 43.92 \\
\hline & Pinus wallichiana & $5 \%$ & $1 \%$ & $1 \%$ & 2.33 & $2^{\text {nd }}$ & 1.30 & 0.01 \\
\hline \multirow{2}{*}{ Stand 19} & Picea smithiana & $87 \%$ & $96 \%$ & $96 \%$ & 93 & $1^{\text {st }}$ & 141.55 & 60.00 \\
\hline & Pinus wallichiana & $13 \%$ & $4 \%$ & $4 \%$ & 7 & $2^{\text {nd }}$ & 5.90 & 0.07 \\
\hline Stand 20 & Picea smithiana & $100 \%$ & $100 \%$ & $100 \%$ & 100 & Pure & 76.42 & 39.30 \\
\hline \multirow{2}{*}{ Stand 21} & Picea smithiana & 62 & 76 & 10 & 76 & $1^{\text {st }}$ & 134.70 & 20.78 \\
\hline & Betula utilis & 38 & 24 & 90 & 24 & $2^{\text {nd }}$ & 42.50 & 0.74 \\
\hline \multirow{2}{*}{ Stand 22} & Picea smithiana & 69 & 85 & 88 & 80.67 & $1^{\text {st }}$ & 100.26 & 32.03 \\
\hline & Pinus wallichiana & 31 & 15 & 12 & 19.33 & $2^{\text {nd }}$ & 17.70 & 0.77 \\
\hline \multirow{2}{*}{ Stand 23} & Picea smithiana & 61 & 73 & 91 & 75 & $1^{\mathrm{st}}$ & 116.06 & 20.30 \\
\hline & Betula utilis & 39 & 27 & 9 & 25 & $2^{\text {nd }}$ & 42.90 & 0.76 \\
\hline Stand 24 & Picea smithiana & 100 & 100 & 100 & 100 & Pure & 201.69 & 42.09 \\
\hline \multirow{2}{*}{ Stand 25} & Picea smithiana & 65 & 81 & 94 & 80 & $1^{\mathrm{st}}$ & 119.70 & 1.76 \\
\hline & Betula utilis & 35 & 19 & 06 & 20 & $2^{\text {nd }}$ & 28.10 & 0.41 \\
\hline
\end{tabular}


Table 3. Continued.

\begin{tabular}{|c|c|c|c|c|c|c|c|c|}
\hline \multirow{3}{*}{ Stand 26 } & Picea smithiana & 71 & 86 & 85 & 80.67 & $1^{\text {st }}$ & 149.87 & 45.38 \\
\cline { 2 - 9 } & Betula utilis & 29 & 14 & 15 & 19.33 & $2^{\text {nd }}$ & 24.40 & 1.35 \\
\hline
\end{tabular}

Key to abbreviations: R. F = Relative Frequency, R.D = Relative density, R.B.A = Relative Basal area, IVI = Importance value Index, D/ha ${ }^{-1}=$ Density/hector of species, $\mathrm{BAm}{ }^{2} \mathrm{ha}^{-1}=$ Basal area of species $\mathrm{m}^{2} /$ hectare, $1^{\text {st }}=$ First dominant species, $2^{\text {nd }}=$ Second dominant species, $3^{\text {rd }}=$ Third dominant species, STD $=$ Stand Number.

Table 4. Summary table of phytosociology of sampled trees species.

\begin{tabular}{|c|c|c|c|c|c|c|c|c|}
\hline \multirow{2}{*}{ S.No } & \multirow{2}{*}{ Spp. Code } & \multirow{2}{*}{ PNST } & \multirow{2}{*}{ Mean IVI } & \multirow{2}{*}{ Mean D ha-1 } & \multirow{2}{*}{ Mean B.A m² ha $^{-1}$} & \multicolumn{3}{|c|}{ Dominant } \\
\hline & & & & & & $1^{\mathrm{st}}$ & $2^{\text {nd }}$ & $3^{\text {rd }}$ \\
\hline 1 & P.W & 18 & $67.68 \pm 10.46$ & $170.6 \pm 30.77$ & $24.86 \pm 5.09$ & 11 & 05 & 0 \\
\hline 2 & P.S & 15 & $79.97 \pm 7.08$ & $108.7 \pm 12.66$ & $26.12 \pm 4.65$ & 13 & 02 & 0 \\
\hline 3 & B.U & 06 & $48.05 \pm 16.45$ & $53.43 \pm 15.37$ & $2.98 \pm 1.68$ & 02 & 04 & 0 \\
\hline 4 & J.E & 01 & $29 \pm 00$ & $82.3 \pm 00$ & $1.66 \pm 00$ & 0 & 01 & 0 \\
\hline
\end{tabular}

Key to Abbreviations: $\pm=$ Standard Error, PNST $=$ Presence in Number of stands D = Density, B.A = Basal area, $\mathrm{Sp}=$ species, $\mathrm{PW}=$ Pinus wallichiana, $\mathrm{P} . \mathrm{S}=$ Picea smithiana, $\mathrm{J} . \mathrm{E}=$ Juniperus excelsa, $\mathrm{B} . \mathrm{U}=$ Betula utilis

Miandam of the Himalayan Range, they recorded that the Pinus wallichiana 96 stems ha $^{-1}$ with $18 \%$ relative basal. Moreover, from different climatic zones of Pakistan and Takht-e-Silaiman (Baluchistan), [38] recorded the densities of Pinus wallichiana 337 stem ha $^{-1}$

Stand No.1

\section{Density $\mathrm{m}^{2} / \mathrm{ha}=60.83$}

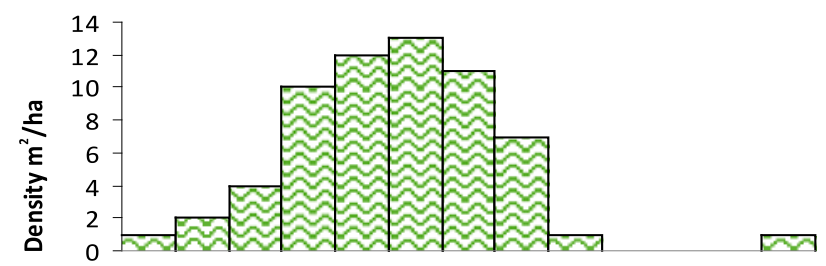

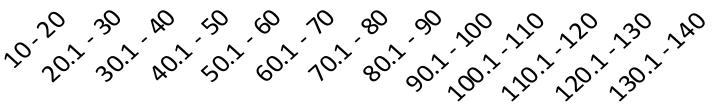

Dbh Size Classes

Stand No.2

Density $\mathrm{m}^{2} / \mathrm{ha}=126.71$

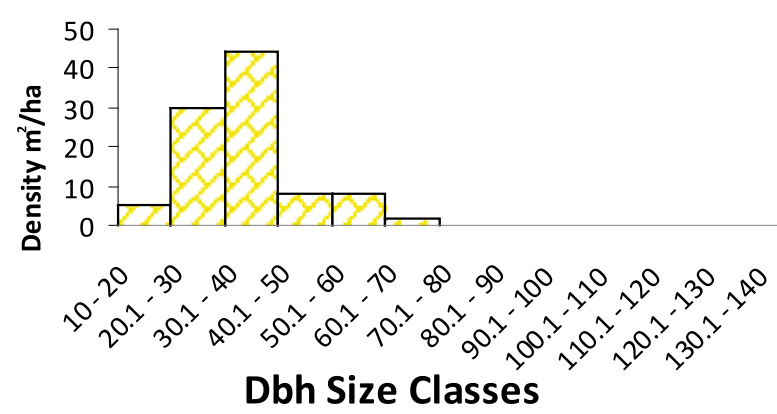

Fig. 1. Size class structure of 26 stands of the study area. and 232 stems ha ${ }^{-1}$ respectively. Himalayan pine also is known as the evergreen Pinus wallichiana tree, which is naturally distributed from Afghanistan across the entire Himalayan region, including Pakistan, India, Nepal and Bhutan, having altitude ranging from 1800-3900 m

\section{Stand No.3 \\ Density $\mathrm{m}^{2} / \mathrm{ha}=93.91$}

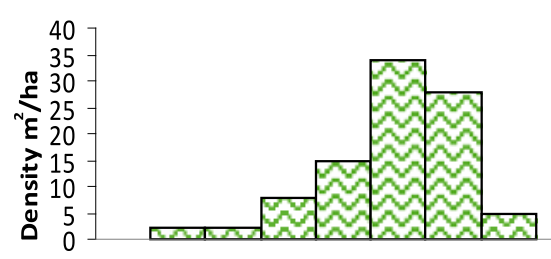

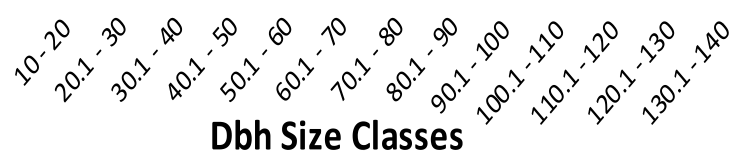

Stand No.4

Density $\mathrm{m}^{2} / \mathrm{ha}=\mathbf{2 3 4 . 2 6}$

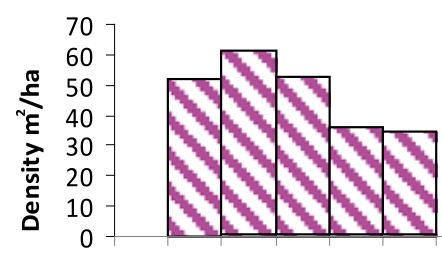

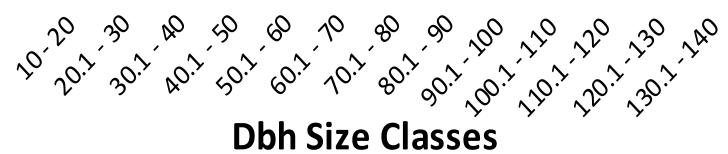




\section{Stand No.5}

Density $\mathrm{m}^{2} / \mathrm{ha}=\mathbf{2 3 2 . 9 9}$

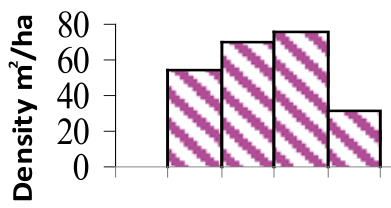

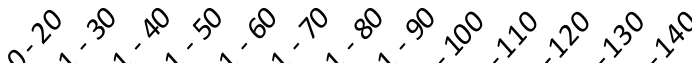

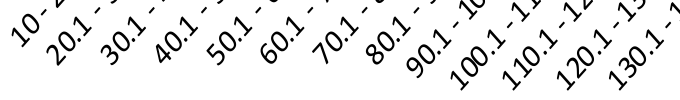

Dbh Size Classes

Stand No.6

Density $\mathrm{m}^{2} / \mathrm{ha}=159.58$

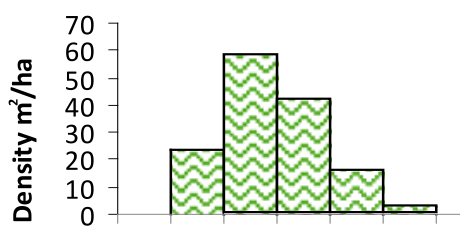

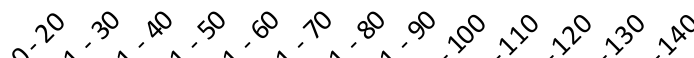

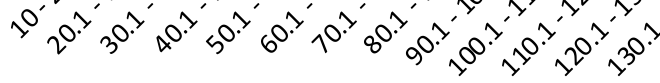

Dbh Size Classes

Stand No.7

Density $\mathrm{m}^{2} / \mathrm{ha}=\mathbf{5 6 . 2 4}$

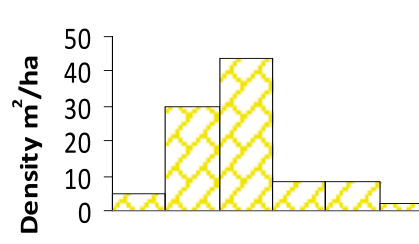

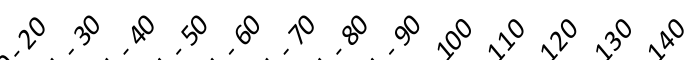

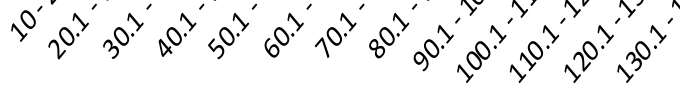

Dbh Size Classes

Stand No.8

Density $\mathrm{m}^{2} / \mathrm{ha}=\mathbf{2 5 8 . 8}$

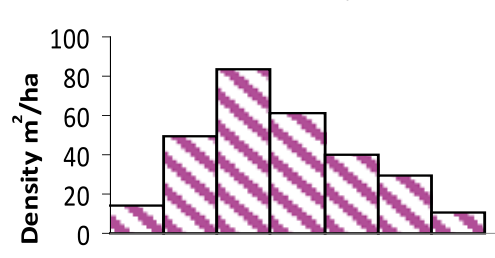

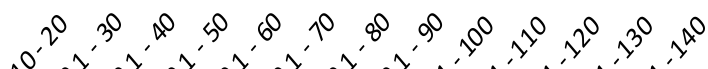

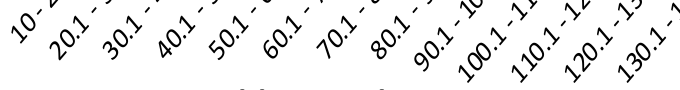

Dbh Size Classes

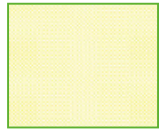

Betula utilis

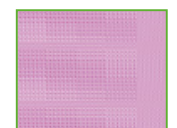

Pinus wallichiana
Stand No.9

Density $\mathrm{m}^{2} / \mathrm{ha}=\mathbf{2 8 6 . 9 4}$

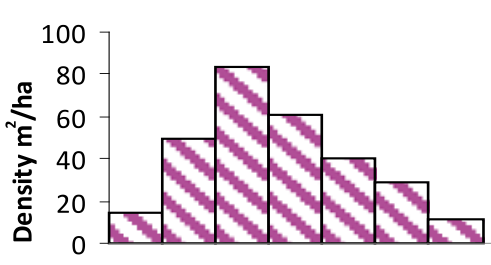

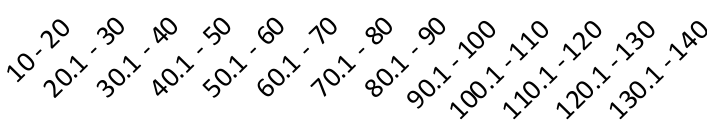

Dbh Size Classes

Stand No.10

Density $\mathrm{m}^{2} / \mathrm{ha}=\mathbf{3 0 7 . 4 1}$

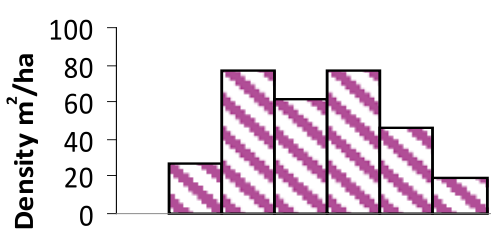

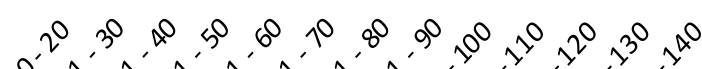

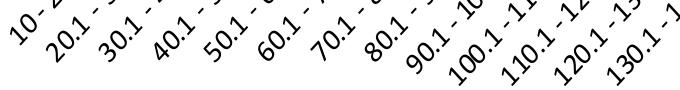

Dbh Size Classes

Stand No.11

Density $\mathrm{m}^{2} / \mathrm{ha}=178.36$

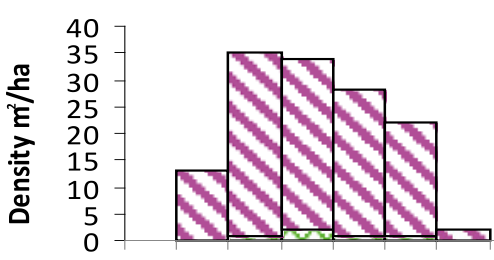

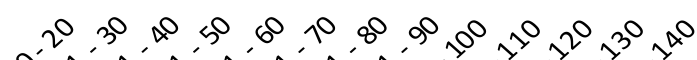

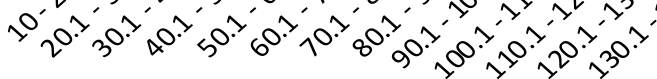

Dbh Size Classes

Stand No.12

Density $\mathrm{m}^{2} / \mathrm{ha}=159.41$

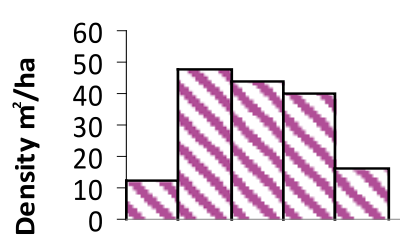

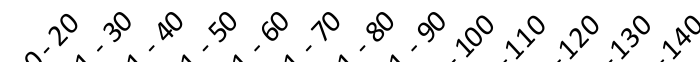

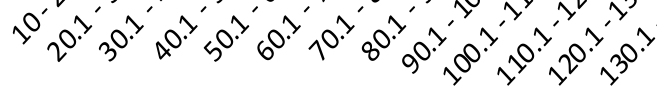

Dbh Size Classes
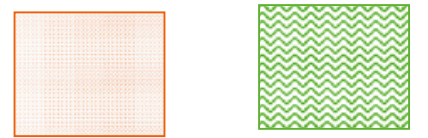

Juniperus excelsa

Picea smithiana

Fig. 1. Continued. 
Stand No.13

Density $\mathrm{m}^{2} / \mathrm{ha}=293.43$

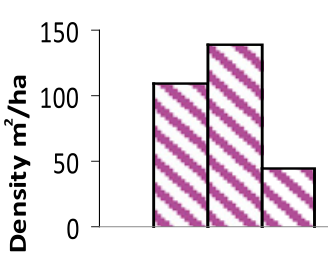

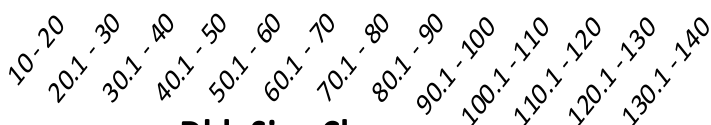

Dbh Size Classes

Stand No.14

Density $\mathrm{m}^{2} / \mathrm{ha}=331.13$

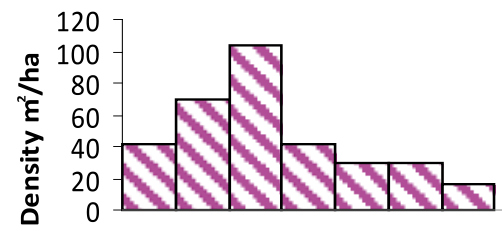

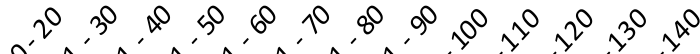

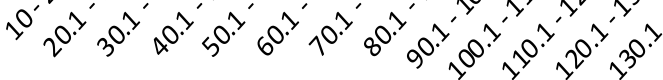

Dbh Size Classes

Stand No.15

Density $\mathrm{m}^{2} / \mathrm{ha}=266.67$

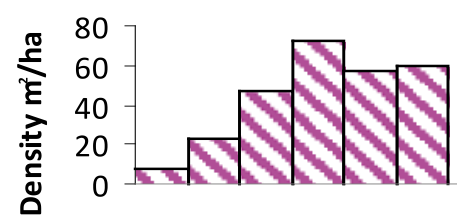

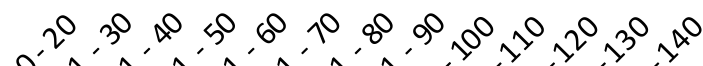

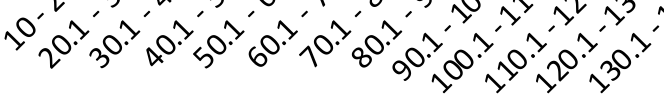

Dbh Size Classes

Stand No.16

Density $\mathrm{m}^{2} / \mathrm{ha}=\mathbf{2 5 7 . 2}$

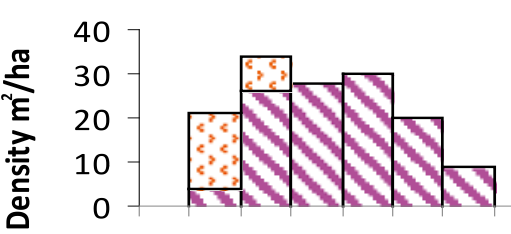

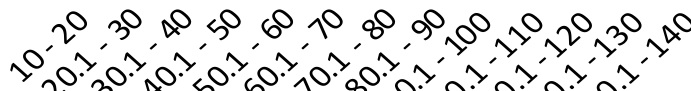

Dbh Size Classes

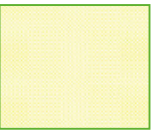

Betula utilis
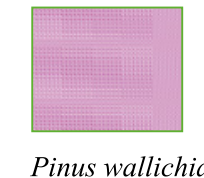

Pinus wallichiana
Stand No.17

Density $\mathrm{m}^{2} / \mathrm{ha}=\mathbf{1 2 9 . 7 7}$

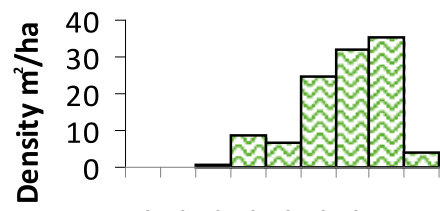

$, 2,3,2,5,6,1,8,9,0,0,0,2,3,0$

20.

Dbh Size Classes

Stand No.18

Density $\mathrm{m}^{2} / \mathrm{ha}=127.88$

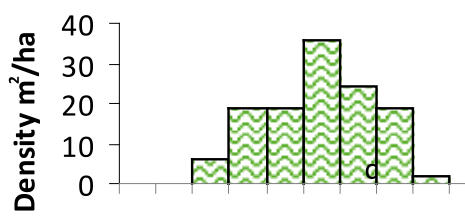

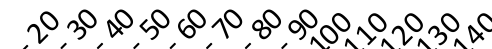

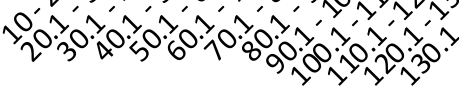

Dbh Size Classes

Stand No.19

Density $\mathrm{m}^{2} / \mathrm{ha}=137.45$

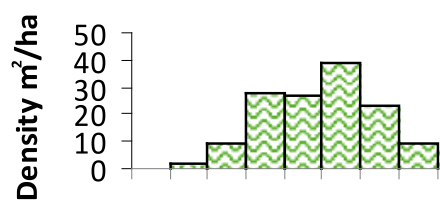

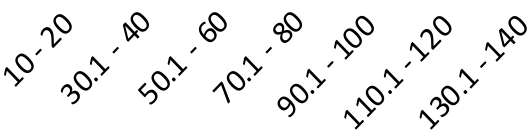

Dbh Size Classes

Stand No.20

Density $\mathrm{m}^{2} / \mathrm{ha}=76.42$

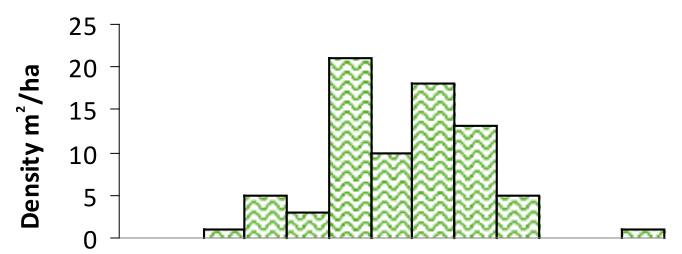

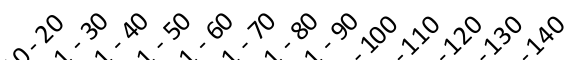

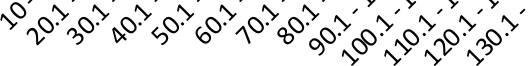

Dbh Size Classes

Fig. 1. Continued.

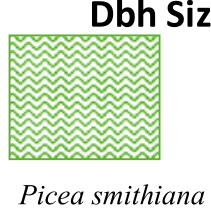




\section{Stand No.21 \\ Density $\mathrm{m}^{2} / \mathrm{ha}=177.2$}

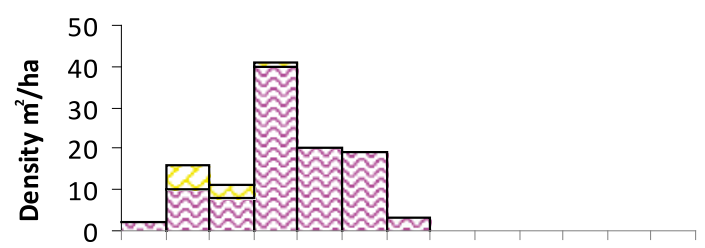

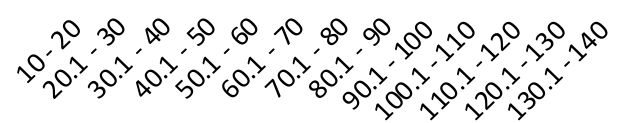

Dbh Size Classes

Stand No.23

Density $\mathrm{m}^{2} / \mathrm{ha}=158.96$

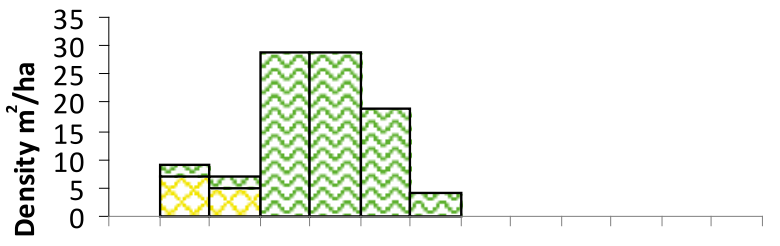

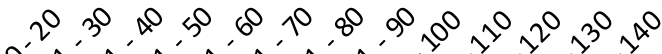

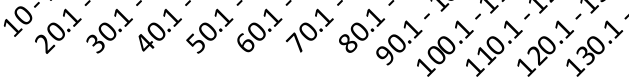

Dbh Size Classes

Stand No.24

Density $\mathrm{m}^{2} / \mathrm{ha}=\mathbf{2 0 1 . 6 9}$

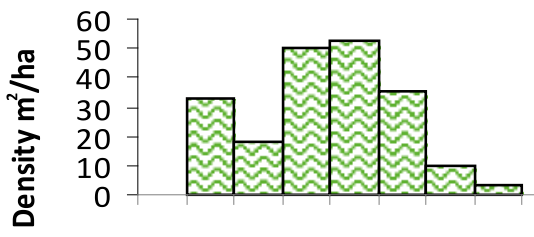

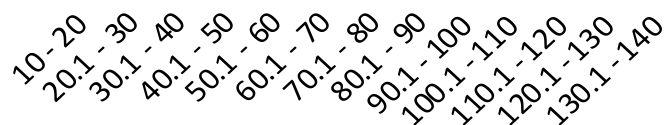

\section{Dbh Size Classes}

Fig. 1. Continued.

[38-40] described that Pinus wallichiana may grow in moist temperate as well as in dry temperate mountainous regions in Pakistan. This shows the full ecological amplitude of this species.

During this study, Picea smithiana tree species were recorded in 15 stands having 108.7 \pm 12.66 mean density $\mathrm{ha}^{-1}$. This species found as $1^{\text {st }}$ leading species in 13 locations and as $2^{\text {nd }}$ leading in two stands (Table 4). Many other scholars also studied Picea smithiana (Wall). Boiss, in different parts of Pakistan, e.g., density 333 stems per hector having 167 basal area $\mathrm{m}^{2}$ from Naltar (Gilgit) forest described by Ahmed et al. [38] and lowest density with 35 individuals ha ${ }^{-1}$ from Afghanistan recorded by Wahab et al. [35]. This huge difference of these forest is due to the large amount

\section{Stand No.25 \\ Density $\mathrm{m}^{2} / \mathrm{ha}=147.8$}

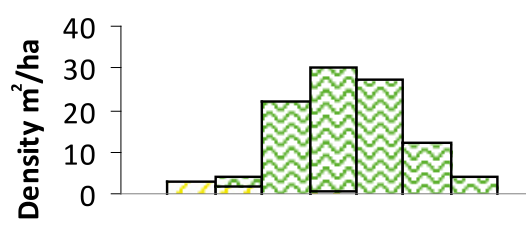

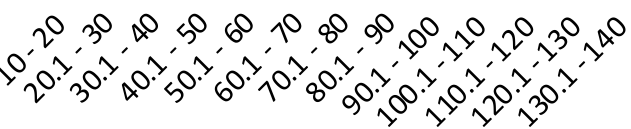

Dbh Size Classes

Stand No.26

Density $\mathrm{m}^{2} / \mathrm{ha}=174.27$

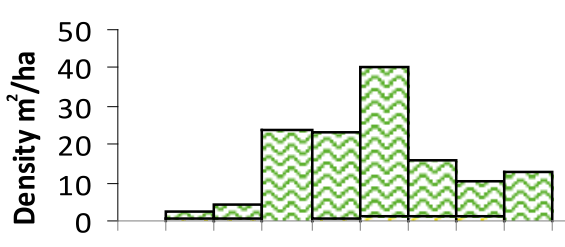

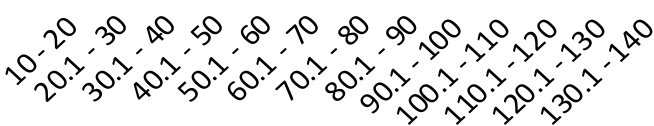

Dbh Size Classes
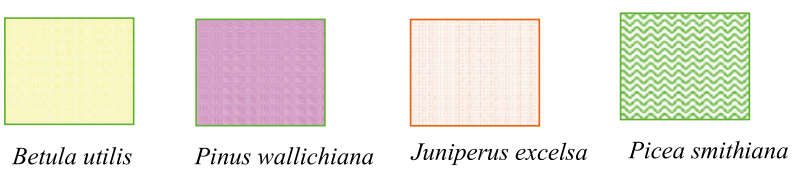

of cutting natural forest and other disturbances in these areas. Human-induced factors, overgrazing of livestock, cutting of timber for domestic needs, and other natural disturbances [41]. Similarly, Akbar et al. [15, 39]

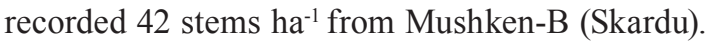

An angiosperm broadleaved tree species, Betula utilis, was observed in only two sites as the $1^{\text {st }}$ dominant species with $53.43 \pm 15.37$ mean density $\mathrm{ha}^{-1}$ in the study area and in 4 stands as the second most-plentiful species (Table 4). Similarly, Betula utilis, a co-dominant species, was recorded by Ahmed et al. [38] from Nalter Valley Gilgit with 666 stems ha-1 and $30 \mathrm{~m}^{2} \mathrm{ha}^{-1}$ basal area. Akbar et al. [15] described that the Betula utilis employed the highest density 159 and 121 stems ha ${ }^{-1}$ from Joglotgah and Gasing Gilgit. 
Among the all stands, Juniperus excelsa appeared in only one stand at Naltar Bala (stand No. 16 in Lower Besha Giri) as the second most-dominant species with 82.30 stems ha-1 $^{-1}$ (Table 4). Akbar et al. [42] also reported that Juniperus excelsa attained with 96 stems ha-1 the highest value from Gasing (Kharmang). The same species was also studied by Ahmed et al. [38] with 175 stems ha-1 and $42 \mathrm{~m}^{2} \mathrm{ha}^{-1}$ basal area from Baluchistan Province.

In this study gaps in early size classes of trees were recorded in 15 locations representing the less regeneration of seedlings, over grazing and cone collection by the inhabitance similar observation noticed by $[8,11,35,42]$ during their studies. Satisfactory individuals found in middle size classes, however, that the futures of the forest are under threat because of improper regeneration of early size classes.

Gaps in large and extra-large size classes were identified in the entire area. There are no single stands without gaps, indicating the server illegal cutting of mature trees in the studied forested area. Gaps in large and extra-large size classes were also observed by [15, $35,38,42-44]$ from the different locations of frosted areas in Pakistan. Diameter size class distribution is used for the better forest management and conservation needs and sustainable utilization in the developing countries frequently [7]. Without analyzing the distribution status of trees, better management and conservation could not be applied in any forest stands. Diameter distribution and the related statistical model can play an important role in some forest discipline, including forestry, and is helpful for the better management practices of forest conservation [45].

\section{Conclusions}

In light of this study, we concluded that gaps in small, middle, large and extra-large classes were observed in different locations representing noticeable disturbances, natural (i.e., sliding, forest fire, flood, soil erosions, earthquakes) and anthropogenic (i.e., illegal cutting, looping, tapering, burning, grazing and ranching). No single stand showed an ideal regeneration pattern, indicating the threat for future trends of these forests. Noticeable gaps in small, large and extra-large classes are also evident for the mentioned categories of disturbances. Introducing social forestry, preventing illegal anthropogenic activities, providing alternative livelihood options for inhabitants and exercising the legislative power form concern authorized departments of these valuable forests could be preserved for the sustainable use for future generations. This study is also helpful to expose the present status and future trends as well as could be utilized for better management and conservation practices in the region, conducting a similar study in the rest of forested areas in GilgitBaltistan.

\section{Conflict of Interest}

The authors declare no conflict of interest.

\section{References}

1. GILLIAM F.S. Forest ecosystems of temperate climatic regions: from ancient use to climate change. New Phytologist, 212 (4), 871, 2016.

2. DE GOUVENAIN R.C., SILANDER J.A. Temperate Forests. Reference Module in Life Sciences, 2, 1, 2017.

3. AFTAB E., HICKEY G.M. Forest administration challenges in Pakistan: the Case of the Patriata Reserved Forest and the "New Murree" Development. The International Forestry Review. Commonwealth Forestry Association. 2010.

4. CETIN M., SEVIK H. Measuring the impact of selected plants on indoor $\mathrm{CO}_{2}$ concentrations. Polish Journal of Environmental Studies, 25 (3), 973, 2016.

5. ZHENG L.F., ZHOU X.N. Diameter distribution of trees in natural stands managed on polycyclic cutting system. Forestry Studies in China, 12 (1), 21, 2010.

6. PODLASKI R. Suitability of the selected statistical distributions for fitting diameter data in distinguished development stages and phases of near-natural mixed forests in the Świętokrzyski National Park (Poland). Forest Ecology and Management, 236 (2-3), 393, 2006.

7. FALLAHCHAI M.M., SHOKRI S. The evaluation of different statistical distributions in order to fit Alnus subcordata C.A.M. species diameter in mountainous forests north of Iran. Biological Forum, 6 (1), 109, Retrieved from http://researchtrend.net/bf12/21... 2014.

8. HUSSAIN A., SHAUKAT S.S., AHMED M., AKBAR M., MAGSI H.Z. Modeling the diameter distribution of gymnosperm species from central Karakoram National Park, Gilgit Baltistan, and Pakistan using weibull function. Journal of biodiversity and environmental science, 5 (1), 330. Retrieved from http://citeseerx.ist.psu.edu/viewdoc/do wnload?doi=10.1.1.652.7529\&rep=rep1\&type $=$ pdf 2014.

9. POND N.C., FROESE R.E. Interpreting Stand Structure through Diameter Distributions. Forest Science, 61 (3), 429, 2015.

10. RUBIN B.D., MANION P.D., FABER-LANGENDOEN D. Diameter distributions and structural sustainability in forests. Forest Ecology and Management, 222 (1-3), 427, 2006.

11. SIDDIQUI M.F., SHAUKAT S.S., AHMED M., KHAN N., KHAN I.A. Vegetation-environment relationship of conifer dominating forests of moist temperate belt of Himalayan and Hindukush Regions of Pakistan. Pakistan Journal of Botany, 45 (2), 577, 2013.

12. PODLASKI R., ROESCH F.A. Modelling diameter distributions of two-cohort forest stands with various proportions of dominant species: A two-component mixture model approach. Mathematical Biosciences, 249 (1), 60, (2014.

13. AUGUSTO R., LIMA F. DE, BATISTA F., INA P., DE LIMA R.A.F., BATISTA J.L.F., PRADO P.I. Modeling Tree Diameter Distributions in Natural Forests: An Evaluation of 10 Statistical Models Renato. Forest Science, 60, 1, 2014.

14. LONSDALE J., MINUNNO F., MENCUCCINI M., PERKS M. Bayesian calibration and Bayesian model 
comparison of a stand level dynamic growth model for Sitka spruce and Scots pine. Forestry, 88 (3), 326, 2014.

15. AKBAR M., SHAUKAT S.S., AHMED M., HUSSAIN A., HYDER S., ALI S., ALI K. Characterization of diameter distribution of some tree species from Gilgit-baltistan using weibull distribution. Journal of Biodiversity and Environmental Sciences J. Bio. \& Env. Sci, 5 (4), 2220, 2014.

16. ROBINSON A.P., HAMANN J.D. Forest analytics with $\mathrm{R}$ : an introduction. Springer. Retrieved from https://www. springer.com/us/book/9781441977618 2011.

17. CETIN M. Chronicles and geoheritage of the ancient Roman city of Pompeiopolis: a landscape plan. Arabian Journal of Geosciences. 2018.

18. CETIN M., SEVIK H., CANTURK U., CAKIR C. Evaluation of the Recreational Potential of Kutahya Urban Forest. Fresenius Environmental Bulletin, 27 (5), 2629, 2018.

19. CETIN M., SEVIK H. Evaluating the recreation potential of Ilgaz Mountain National Park in Turkey. Environmental Monitoring and Assessment, 188 (1), 1, 2016.

20. CETIN M., ZEREN I., SEVIK H., CAKIR C., AKPINAR H. A study on the determination of the natural park's sustainable tourism potential. Environmental Monitoring and Assessment, 190 (3), 2018.

21. YUCEDAG C., KAYA L.G., CETIN M. Identifying and assessing environmental awareness of hotel and restaurant employees' attitudes in the Amasra District of Bartin. Environmental Monitoring and Assessment, 190 (2), 2018.

22. CETIN M., SEVIK H. Assessing Potential Areas of Ecotourism through a Case Study in Ilgaz Mountain National Park. In Tourism - From Empirical Research Towards Practical Application. InTech. 2016.

23. CETIN M. Evaluation of the sustainable tourism potential of a protected area for landscape planning: A case study of the ancient city of Pompeipolis in Kastamonu. International Journal of Sustainable Development and World Ecology, 22 (6), 490, 2015.

24. KUSCU I.S.K., CETIN M., YIGIT N., SAVACI G., SEVIK H. Relationship between enzyme activity (Ureasecatalase) and nutrient element in soil use. Polish Journal of Environmental Studies, 27 (5), 2107, 2018.

25. TURKYILMAZ A., SEVIK H., CETIN M., AHMAIDA SALEH E.A. Changes in heavy metal accumulation depending on traffic density in some landscape plants. Polish Journal of Environmental Studies, 27 (5), 2277, 2018.

26. KRAVKAZ-KUSCU I.S., SARIYILDIZ T., CETIN M., YIGIT N., SEVIK H., SAVACI G. Evaluation of the soil properties and primary forest tree species in Taskopru (Kastamonu) district. Fresenius Environmental Bulletin, 27 (3), 1613, 2018.

27. CETIN M. Sustainability of urban coastal area management: A case study on Cide. Journal of Sustainable Forestry, 35 (7), 527, 2016.

28. KAYA E., AGCA M., ADIGUZEL F., CETIN M. Spatial data analysis with $\mathrm{R}$ programming for environment. Human and Ecological Risk Assessment: An International Journal, 0 (0), 1, 2018.

29. CETIN M. Using GIS analysis to assess urban green space in terms of accessibility: Case study in Kutahya. International Journal of Sustainable Development and World Ecology, 22 (5), 420, 2015.
30. CETIN M. Determining the bioclimatic comfort in Kastamonu City. Environmental Monitoring and Assessment, 187 (10), 2015.

31. CETIN M., ADIGUZEL F., KAYA O., SAHAP A. Mapping of bioclimatic comfort for potential planning using GIS in Aydin. Environment, Development and Sustainability, 20 (1), 361, 2018.

32. COTTAM G., CURTIS J.T. The Use of Distance Measures in Phytosociological Sampling. Ecological Society of America, 37(3), 451. Retrieved from http://cescos.fau.edu/ gawliklab/papers/CottamGandJTCurtis1956.pdf 1956.

33. AHMED M., SHAUKAT S.S. A text book of vegetation ecology. Abrar Sons. Retrieved from https://books.google. co.uk/books/about/A_Text_Book_of_Vegetation_Ecology. html?id=1uC2jwEACAAJ\&redir_esc=y 2012.

34. NASIR E., ALI I.S. Flora of Pakistan. National Herbarium, NARC, Islamabad, Department of Botany, University of Karachi, Karachi (Fascicles). 1972.

35. WAHAB M. Population dynamics and dendrochronological potential of pine tree species of District Dir Pakistan. Retrieved from https://scholar.google.es/scholar?cluster= $2333103418807388141 \&$ hl $=$ en\&as_sdt $=2005 \&$ sciodt $=0,5$ 2011.

36. MUELLER-DOMBOIS D., ELLENBERG H. Aims and methods of vegetation ecology. Wiley. Retrieved from https://books.google.co.uk/books/ about/Aims_and_Methods_of_Vegetation_Ecology. html?id=amFKT9AEQiUC\&redir_esc=y 1974.

37. AHMED M., NAQVI S.H. Tree-ring chronologies of Picea smithiana (Wall.) Boiss., and its quantitative vegetational description from Himalayan range of Pakistan. Pakistan Journal of Botany, 37 (3), 697, 2005.

38. AHMED M., HUSAIN T., SHEIKH A. H., SADRUDDIN S., MUHAMMAD H., SIDDIQUI F., AL E.T. Phytosociology and Structure of Himalayan Forests From Different Climatic Zones of Pakistan. Pakistan Journal of Botany, 38 (2), 361, 2006.

39. AKBAR M., KHAN H., HUSSAIN A., HYDER S., BEGUM F., KHAN M., ALI S. Present status and future trend of chilghoza forest in Department of Environmental Sciences Karakoram International University GilgitBaltistan , Department of Biological Sciences Karakoram International University Gilgit-Baltistan, Pakistan. J. Bio. \& Env. Sci., 5 (5), 253, 2014.

40. SHAHEEN H., SHINWARI Z.K. Phytodiversity and endemic richness of Karambar lake vegetation from Chitral, Hindukush-Himalayas. Pakistan Journal of Botany, 44 (1), 15. Retrieved from http://www.pakbs.org/ pjbot/PDFs/44(1)/03.pdf 2012.

41. CHAMPION H. Forest types of Pakistan. [Peshawar]: [Pakistan Forest Institute]. Retrieved from http://www. worldcat.org/title/forest-types-of-pakistan/oclc/402929 1965.

42. AKBAR M., AHMED M., HUSSAIN F., SIDDIQUI M. F., RAZA G., HYDER S., ABBAS N. Relationship of forest vegetation and environmental gradients (Adaphic, topographic and soil nutrients) from some frosted areas of himalayan, hindu kush and karakoram ranges of GilgitBaltsitan, Pakistan- (a multivariate approach). Pakistan Journal of Botany, 49 (Special Issue), 255, 2017.

43. AKBAR M., AHMED M., SHAHID SHAUKAT S., HUSSAIN A., ZAFAR M.U., SARANGZAI A.M., HUSSAIN F. Size Class Strucure of Some Forests From Himalayan Range of Gilgit-Baltistan. Sci., Tech. and Dev, 32 (1), 56, 2013. 
44. HUSSAIN A., FAROOQ M.A., AHMED M., ZAFAR M. U., AKBER M. Phytosociology and structure of Central Karakuram National Park (CKNP) of Northern areas of Pakistan. world Applied Science J., 9 (1), 1443, 2010.
45. SGHAIER T., CAÑELLAS I., CALAMA R., SÁNCHEZGONZÁLEZ M. Modelling diameter distribution of Tetraclinis articulata in Tunisia using normal and weibull distributions with parameters depending on stand variables. iForest Biogeosciences and Forestry, 9 (5), 702, 2016. 